La (des)aparición de los monumentos. Materialidad e imaginarios en el panóptico de lbagué, Colombia

Fabián Andrés Sánchez Rojas - Andrés Ernesto Francel Delgado

páginas / año 12 - n 29 Mayo-Agosto / ISSN 1851-992X/ 2020

http://revistapaginas.unr.edu.ar/index.php/RevPaginas

\title{
La (des)aparición de los monumentos. Materialidad e imaginarios en el panóptico de Ibagué, Colombia
}

\author{
The (dis)appearance of monuments. Materiality and imaginaries in the \\ Ibague panopticon, Colombia
}

\author{
Fabián Andrés Sánchez Rojas \\ fabiansanchez1979@hotmail.com \\ Universidad del Tolima (Colombia)
}

Andrés Ernesto Francel Delgado

Departamento de Arquitectura y Diseño, grupo de investigación E-ARC, Universidad del Tolima (Colombia) aefranceld@ut.edu.co

\begin{abstract}
Resumen
El panóptico de Ibagué es un bien de interés cultural de carácter nacional que ha decaído en estado ruinoso desde el año 2003, como consecuencia de un plan de restauración mediado por las deficiencias administrativas gubernamentales. Para estudiar su impacto en la sociedad, se abordó un estudio alrededor de los imaginarios de identidad, cultura y patrimonio asociados al panóptico, para lo cual se implementó un cuestionario y se contrastaron sus datos con diversas fuentes documentales en una dimensión histórica. La (des)aparición observada consiste en la disyuntiva entre la liberación del edificio por la demolición de sus muros perimetrales, con lo cual se inserta en las dinámicas urbanas -o aparición- y, simultáneamente, comienza un proceso de degradación física y simbólica -o desaparición-. Se concluye con la necesidad de que los procesos de intervención del patrimonio material se basen en los estudios sobre la identidad de los pueblos para su puesta en valor y legitimación, para evitar que los dispositivos arquitectónicos se conviertan en arbitrariedades particulares y agendas políticas.
\end{abstract}

Palabras clave

Patrimonio; identidad; restauración; conservación; panóptico

\section{Abstract}

The Ibague panopticon is a National Heritage Site that has declined in a ruined condition since 2003 , as a result of a restoration plan mediated by government administrative deficiencies. To study its impact on society, a study was carried out around the imaginary of identity, culture and heritage associated with the panopticon, for which a questionnaire was implemented and its data was compared with various documentary sources in a historical dimension. The (dis) appearance observed consists of the trade-off between the liberation of the building by the demolition of its perimeter walls, thereby inserting itself into urban

Esta obra está sujeta a la Licencia Reconocimiento-NoComercial-CompartirIgual 4.0 Internacional de Creative Commons. http://creativecommons.org/licenses/by-nc-sa/4.0/ 
dynamics -or appearance- and, simultaneously, a process of physical and symbolic degradation begins -or disappearance-. It concludes with the need for the processes of intervention of the material heritage to be based on studies on the identity of the peoples for their enhancement and legitimation, to prevent architectural devices from becoming particular arbitrariness and political agendas.

Keywords

Heritage, identity, restoration, conservation, panopticon

\section{Introducción}

El Panóptico de Ibagué ha sido estudiado desde sus componentes materiales e históricos, que conducen a exaltar las concepciones espaciales, las calidades constructivas y la importancia histórica de su creación a finales del siglo XIX, durante la configuración de la división político-administrativa nacional actual. A partir de su intervención, con el propósito de recuperar las instalaciones mediante la conservación, restitución y refuncionalización, los medios de comunicación han documentado y denunciado el deterioro constante de las instalaciones, y las entidades de control han adelantado investigaciones sobre las irregularidades administrativas y financieras de la ejecución del proyecto.

Sin embargo, se identificó que al hablar del panóptico como bien de interés cultural, no se han abordado los impactos en los imaginarios de la población de Ibagué, entendidos como el uso e interiorización de los espacios y sus respectivas vivencias, por parte de unos ciudadanos dentro de su intercomunicación social (Silva, 2006), en ese sistema en el que "las percepciones se transforman en representaciones y éstas, por un proceso simbólico se constituyen en imaginarios" (Lindón, 2007). Por este motivo, se decidió abordar una investigación que tomara en cuenta la apropiación de los habitantes con respecto al panóptico, tanto en el pasado, como en el presente y el futuro. De este modo, se abordó una investigación sobre la visión de los ciudadanos, sus grados de conocimiento sobre la historia de la edificación, sus opiniones sobre los hechos y las expectativas sobre el futuro del bien de interés cultural.

Para abordarlo, se diseñó una encuesta que contuviera datos esenciales para identificar las relaciones que establecen los pobladores con el panóptico y los grados de acierto en las decisiones que toman los proyectistas en arquitectura, con respecto a las realidades sociales. En este sentido, a pesar de la tendencia contemporánea a realizar procesos de diseño participativo, se encuentra que las decisiones en procesos de gran carga simbólica, como la intervención de una cárcel, para resignificarla en un centro cultural, siguen siendo tomadas de arriba hacia abajo, es decir, desde grupos de expertos que determinan la condición de lo patrimonial, sin tomar en cuenta el sustento e impacto socio-cultural que ello implica. Se abordan, por lo tanto, las problemáticas en torno a las tensiones en la legitimación de lo patrimonial y la escasa consideración de la identidad construida por parte de los 


\section{La (des)aparición de los monumentos. Materialidad e imaginarios en el panóptico de Ibagué, Colombia}

habitantes para la puesta en valor de ciertos edificios, en este caso el panóptico, y los múltiples factores que pueden intervenir en ese proceso de legitimación.

El trabajo se aborda desde el ámbito de la historia de la arquitectura y el urbanismo, en el marco de las relaciones establecidas por la maestría en territorio, conflicto y cultura. En este sentido, se reconoce que los ciudadanos construyen imaginarios de cada una las piezas y fragmentos de la ciudad, y estos imaginarios se transforman históricamente, tanto como se rehace la ciudad y la vida urbana que ella aloja (Lindón, 2007). Por lo tanto, el propósito de la investigación consistió en identificar los imaginarios individuales y colectivos, entendidos como verdades profundas de los habitantes (Hiernaux, 2006; Silva, 2006) a partir de los cuales se puede hacer referencia al panóptico, tanto cualitativa como cuantitativamente.

\section{Estado del Arte}

El panóptico de Ibagué fue construido entre 1891 y 1893, como la cárcel principal del departamento del Tolima, aunque sus adecuaciones se prolongaron hasta 1904 (Izasa Nieto, 2015). En 1998 fue declarado Monumento Nacional (Ministro de Cultura de Colombia, 1998) y fue entregado oficialmente por el Instituto Nacional Penitenciario y Carcelario (Inpec) a la gobernación del Tolima, el 30 de mayo de 2003 para realizar las labores de restauración junto al Ministerio de Cultura (Redacción El Tiempo, 2003). En el año 2004, el parlamento andino declaró a Ibagué capital andina de los de los derechos humanos y la paz (Alcaldía municipal de Ibagué, 2004), por lo que el proyecto de intervención del panóptico se enfocó hacia su conversión en museo de los derechos humanos y la paz, cuya inauguración ha sido constantemente aplazada desde 2006, mediada por múltiples eventos de corrupción (Restrepo, 2008; Físcalia General de la Nación secional Tolima, 2012; Ecos del Combeima, 2019).

Origen

El edificio se ubicó originalmente en la zona rural del noroccidente de la ciudad, separado del centro por la quebrada de Los Piojos, con lo cual se aseguraba la protección mutua de las dinámicas carcelarias y las urbanas. Su modelo espacial tomó como base el concepto de panóptico enunciado por Jeremy Bentham, como un dispositivo de control y transformación social mediante la observación de los reclusos desde una garita central sin que el vigilante pueda ser observado, y sin que el recluso sepa si lo observan, pero con la seguridad de que siempre puede ser vigilado (Foucault, 2002).

El modelo de Bentham fue implementado por primera vez en Colombia en el panóptico de Bogotá, diseñado por el arquitecto Thomas Reed, quien fue convocado por el gobierno nacional en 1846 para diseñar el Capitolio Nacional y educar a la primera generación de arquitectos locales (Arango, 1989). El panóptico de Ibagué fue diseñado por el ingeniero Mirtiliano Sicard y construido por los ingenieros Ricardo Correa y Lisandro Herrán (Isaza Nieto, 2015), con el modelo de Reed, a una escala menor, en consecuencia con las limitaciones presupuestales y las determinaciones del gobierno departamental (Francel \& Ojeda, A\&U, 2016). 
En 1921 comenzó el proceso de urbanización de la hacienda en la que había sido construido el panóptico. A su alrededor se desarrolló el barrio Belén, como un barrio parque (Francel, 2015), correspondiente a la implementación de los conceptos de la ciudad jardín para las élites locales (Gómez, 2015). Su diseño estuvo a cargo de los reconocidos arquitectos Alberto Manrique Martin, Arturo Jaramillo Concha y Carlos Arturo Tapia (Francel, 2015), cuyas obras son parte del patrimonio arquitectónico y urbanístico nacional (Pieto Páez, y otros, 2017; El Tiempo, 2010).

Con la creación de Belén, el panóptico quedó inserto en un espacio barrial que rechazaba su uso por la amenaza que representaban las posibilidades de fuga de reclusos. Como consecuencia, el cerramiento perimetral se transformó. Inicialmente fue una reja tras la que se ubicaban jardines, luego se tapiaron los espacios entre las columnas bajas para conformar un muro sólido que incrementó su altura sobre las construcciones aledañas e incorporó nuevas garitas y dependencias para controlar la sobrepoblación de detenidos [Figura 1].

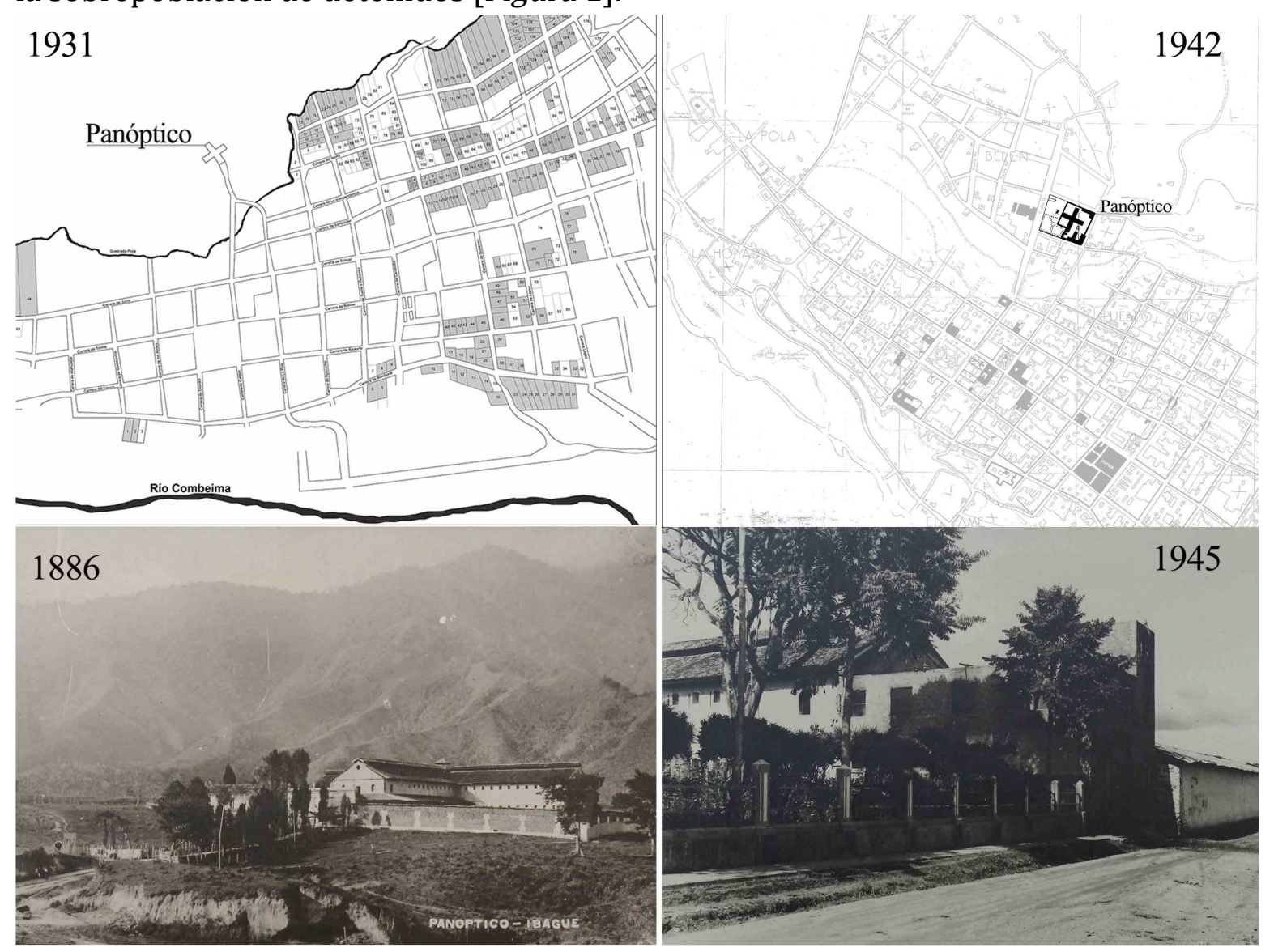

Figura 1. Localización del Panóptico de Ibagué, características de su aislamiento y relaciones con su entorno. Arriba a la izquierda: detalle del "plano de los solares del municipio vendidos entre 1830 y 1930", digitalización propia (2015), fuente: Archivo Histórico Municipal. Arriba a la derecha: plano de la ciudad de Ibagué 1942 fuente: Instituto Geográfico Agustín Codazzi. Abajo a la izquierda: "panóptico de Ibagué", s.a. (1886). Abajo a la derecha: "Jardines del panóptico", fotografía de Eduardo Camocho Ponce (1945), fuente: Archivo de memoria visual del centro cultural Darío Echandía del Banco de la República. 


\section{La (des)aparición de los monumentos. Materialidad e imaginarios en el panóptico de Ibagué, Colombia}

\section{La aparición}

A pesar de ser declarado Monumento Nacional en 1998, fue necesario esperar hasta el año 2006 para que los muros perimetrales se demolieran y el panóptico apareciera, luego de 113 años de estar separado de las dinámicas urbanas. La ciudadanía comenzó un proceso de resignificación de la edificación como objeto material valioso, a través de la apropiación del concepto de panóptico, que constituyó una novedad en el léxico local, pues tradicionalmente se le denominó penal (como dispositivo carcelario del sistema penal), al punto que aún se conserva la marca comercial almojábanas del penal, en un local de comidas situado frente al ingreso de la antigua cárcel. El cambio de denominación de penal a panóptico implicó la transformación del rechazo hacia las palabras cárcel e inseguridad, por la valoración de las palabras panóptico y patrimonio (Restrepo, 2008). El Panóptico de Ibagué, que era invisible, deslumbró y desencadenó proyectos de investigación histórica (Cifuentes Segovia, 2015; Rivera, 2012), de restauración (Pedraza Vargas, Urueña Silva, \& Zuluaga Duque, 2018; Pulido García \& Lovera Bernal, 2018) y de promoción como una de las principales riquezas turísticas de la ciudad, enfocando el patrimonio a su utilidad dentro del sistema de mercado (Vallejo, 2018).

La expectativa que se creó con la recuperación y restauración del Panóptico de Ibagué fue inmensa, así como los recursos destinados para tal fin. La Alcaldía de Ibagué, la Gobernación del Tolima, el Ministerio de Cultura, la clase parlamentaria y los principales actores socioeconómicos, políticos y culturales, aunaron esfuerzos para obtener los recursos necesarios para la restauración, conservación y fomento de la edificación que había sido declarada bien de interés cultural de carácter nacional.

En una primera inversión de recursos en 2001, el Ministerio de Cultura destina $\$ 4.500$ millones (U\$1'964.207) para los estudios y ejecución del proyecto de restauración y recuperación de la edificación, los cuales se ejecutan mediante convenios interadministrativos entre el Ministerio y la Gobernación del Tolima. En 2009, la Alcaldía de Ibagué y la Gobernación del Tolima destinan \$1.600 millones (U\$ 782.778) más para las áreas nuevas del Panóptico (Calero Polanco, Jessica Tatiana; García, Catalina, 2014). Sin embargo, el proyecto devino en un mecanismo de corrupción que ascendió a \$10.000 millones (U\$ 3’044.140) del presupuesto público, de modo que la propuesta de intervención para convertirlo en un dispositivo de la memoria local, de turismo cultural, y símbolo de la riqueza arquitectónica de la ciudad, se constituyó en un elemento de desidia administrativa a todos los niveles gubernamentales [Figura 2]. 


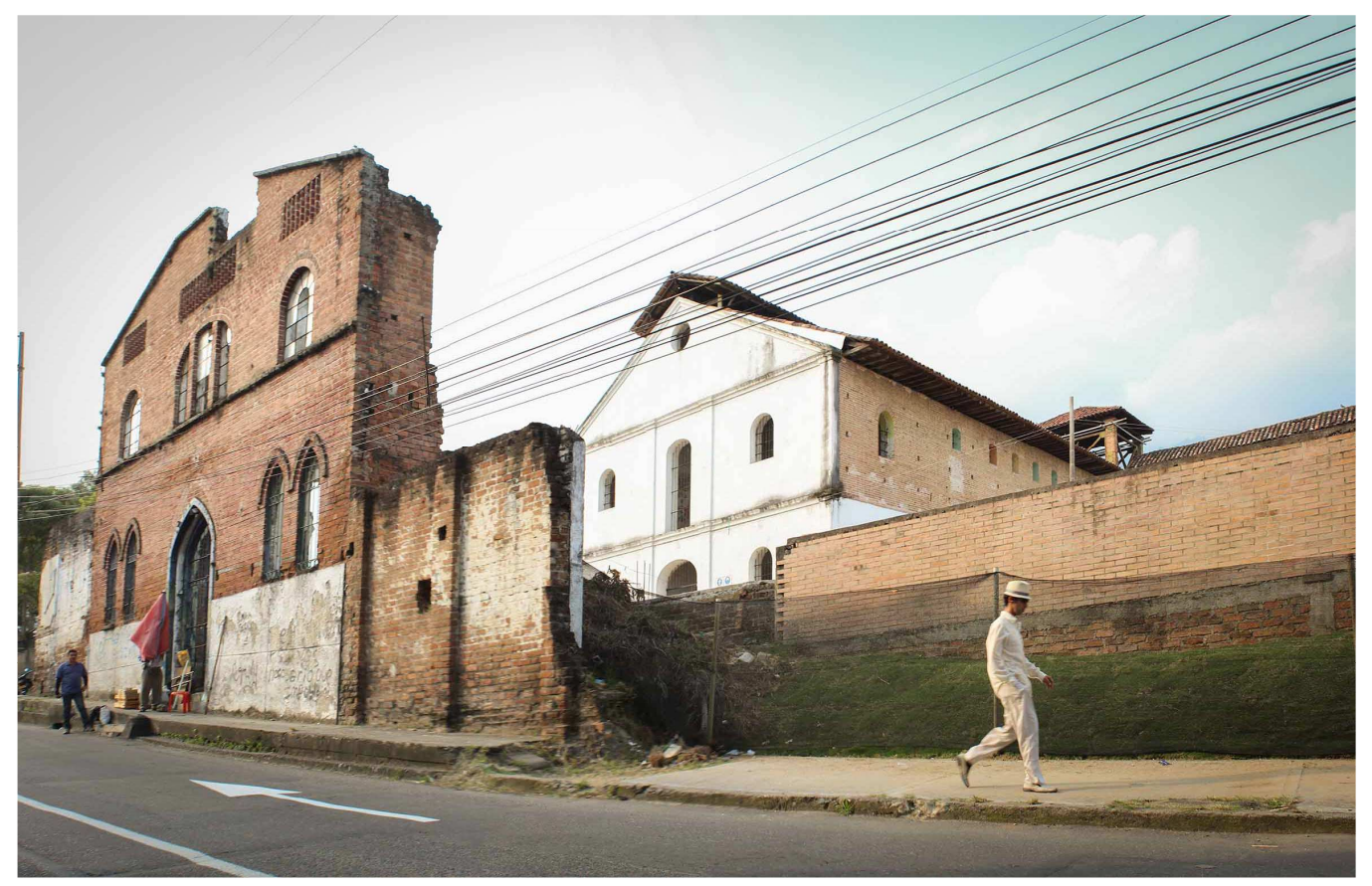

Figura 2. Liberación y ruina del panóptico. Fotografía de José Alejandro Ojeda (2015). Fuente: Grupo de Investigación E-ARC (Estudios de Arquitectura y Ciudad).

\section{Metodología}

Se abordó un método mixto, consistente en una fase de búsqueda documental, principalmente en publicaciones periodísticas y académicas, para recomponer el contexto de desarrollo del panóptico a nivel histórico y del fenómeno de abandono derivado de su intervención. Posteriormente, se diseñó una encuesta como instrumento de captura de datos para reconocer las consecuencias de la aparición material del panóptico.

Se aplicó el enfoque mixto cuali-cuantitativo con el cual se integran distintas técnicas en un mismo estudio. De este modo, se realizaron entrevistas y encuestas "para saber las opiniones de cada cual sobre el tema en cuestión" (Creswell, 2008, pág. 45), con la valoración en escalas numéricas. Las encuestas buscan una perfilación de la población mediante la determinación del rango de edad, escolaridad y años de residencia en la ciudad. Se indaga sobre el conocimiento general de los ciudadanos frente a la edificación, las problemáticas que afectaron el inmueble $y$, finalmente, se cuestiona sobre el futuro del bien como referente de la identidad local.

Para el grado de aproximación a la representatividad estructural se estableció un muestreo por contexto, debido a que, primero, el universo seleccionado es bastante amplio (500.000 habitantes); segundo, que el objeto de investigación es un bien de interés cultural (BIC) en relación con el concepto de identidad; y tercero, que parte de los resultados de la investigación están en función de la apropiación que generaciones futuras realicen del Panóptico. 


\section{La (des)aparición de los monumentos. Materialidad e imaginarios en el panóptico de Ibagué, Colombia}

El muestreo por contexto es una variable del muestreo por juicio y consiste en la selección de las unidades de análisis a partir de criterios conceptuales representativos, o variables que delimitan la composición estructural de la muestra. A ello se articula una aproximación cuantitativa al universo de análisis mediante el uso de censos, padrones, encuestas por cuestionario o cualquier forma de material estadístico (Mejía Navarrete, 2000, pág. 165). En consecuencia, las unidades de análisis son cada una de las 13 comunas en las que se divide la zona urbana de Ibagué a nivel político-administrativo, que corresponden al 95\% de la población municipal (Departamento Administrativo Nacional de Estadística DANE, 2016).

Para seleccionar las comunas como unidades de análisis, se atendió al cumplimiento de las siguientes condiciones: que fueran las más significativas y productivas empíricamente, que pudieran identificarse de manera eficiente y fiable, y que satisficieran los requisitos de las técnicas disponibles (Krippendorff, 1990). Además, que estuvieran claramente definidas en un protocolo de investigación, que en este caso se asume como la división político administrativa vigente (Concejo Municipal de Ibagué, 2018; Concejo Municipal de Ibagué, 2011).

En cuanto a la determinación del tamaño de la muestra, intervienen tres factores: 1) la capacidad operativa de recolección y análisis (el número de casos que podemos manejar de manera realista y de acuerdo con los recursos que dispongamos); 2) el entendimiento del fenómeno (el número de casos que nos permitan responder a las preguntas de investigación, sin que exista una "saturación de categorías"); y 3) la naturaleza del fenómeno bajo análisis (si los casos son frecuentes y accesibles o no, si el recolectar información sobre estos lleva relativamente poco o mucho tiempo) (Hernández Sampieri, 2006).

Luego de determinar como unidad de análisis cada una de las 13 comunas en que se divide la zona urbana de Ibagué, de acuerdo al tipo de estudio de grupos de enfoque, se aplicaron 20 encuestas por comuna, para un total de 260 encuestados, que corresponden a 7 o 10 casos por grupo, de acuerdo con la Tabla 1 de Tamaños de muestra comunes en estudios cualitativos (Hernández Sampieri, 2006) [¡Error! No se encuentra el origen de la referencia.].

Tabla 1. Tamaños de muestra comunes en estudios cualitativos (Hernández Sampieri, 2006).

\begin{tabular}{|l|l|}
\hline Tipo de estudio & Tamaño de muestra sugerido \\
\hline $\begin{array}{l}\text { Etnográfico, teoría fundamentada, } \\
\text { entrevistas, observaciones. }\end{array}$ & 30 a 50 casos. \\
\hline Historia de vida familiar. & $\begin{array}{l}\text { Toda la familia, cada miembro es un } \\
\text { caso. }\end{array}$ \\
\hline Biografía. & $\begin{array}{l}\text { El sujeto de estudio (si vive) y el mayor } \\
\text { número de personas vinculadas a él, } \\
\text { incluyendo críticos. }\end{array}$ \\
\hline Estudios de caso en profundidad. & 6 a 10 casos. \\
\hline
\end{tabular}


Para profundizar en los cuatro grupos de población sugeridos por Hernández Sampieri (2006), se enfatizó la distribución por rangos de edades, coincidente con la pirámide poblacional para la ciudad de Ibagué. Así, un promedio de 62 personas entre los primeros cuatro rangos de edades contestaron el cuestionario, más 21 personas de más de 60 años.

Una vez definidas las unidades de análisis, se procedió a establecer el cuestionario como instrumento de recolección de información, con una primera parte de preguntas imprescindibles para la perfilación del encuestado, como género, edad, nivel socioeconómico, estado civil, escolaridad, barrio o zona de residencia, años de permanencia, pertenencia a agrupaciones y ocupación (Hernández Sampieri, 2006). La segunda parte determina el conocimiento sobre el Panóptico de Ibagué, otras edificaciones patrimoniales y elementos del imaginario colectivo que hacen parte de la identidad de los ciudadanos, opiniones sobre los problemas que ha tenido el Bien de Interés Cultural y, finalmente, cómo ven el futuro de la edificación, una vez sea entregado a la ciudadanía.

Las encuestas se aplicaron aleatoriamente entre el 4 y el 28 de septiembre de 2017 en las 13 comunas, de manera presencial, a transeúntes de 104 barrios, que corresponden al 14,79\% de los 703 barrios de la ciudad. Se tabularon las respuestas y se graficaron sus resultados. Se cruzaron algunas respuestas para determinar la incidencia de variables como rangos de edad, años de residencia en la ciudad y comuna de residencia, que se consideraron pertinentes por el objeto de estudio. Las partes de la encuesta, con sus respectivas partes, gráficos y cruces de variables, se encuentran en el Anexo 1.

\section{Resultados}

El resultado interpretativo de los datos obtenidos en las encuestas, origina el título del artículo, y consiste en la siguiente correlación. El panóptico fue un lugar aislado desde su construcción. A su alrededor creció el barrio Belén, que rechazaba el uso carcelario y desconocía el valor patrimonial del inmueble. Cuando el panóptico fue liberado de los muros perimetrales que le separaban del barrio Belén, apareció físicamente, porque pudo ser observado más allá de sus antiguos límites, como un edificio singular que había estado oculto por su función carcelaria precedente. Simultáneamente a su valoración patrimonial, comenzó un proceso de rechazo, o degradación de su imagen como símbolo de corrupción, por lo cual la mayoría de la población niega su importancia como elemento constitutivo de la identidad local. Sin embargo, existe una ilusión latente por la terminación del edificio, luego de lo cual la mayoría de los encuestados concuerdan en que sería un elemento importante de identidad de los ibaguereños. Esta aparente contradicción presenta la 


\section{La (des)aparición de los monumentos. Materialidad e imaginarios en el panóptico de Ibagué, Colombia}

construcción de imaginarios en relación directa con la condición material del edificio, que funciona como un dispositivo de representación de la corrupción administrativa, tras la que subyace el valor patrimonial, en un esquema de superposición de imaginarios (Gravano, 2005).

El concepto de imaginario es definido como "la inevitable re-presentación, la facultad de simbolización de la cual emergen continuamente todos los miedos, todas las esperanzas y sus frutos culturales" (Durand, 1994). En el panóptico pueden apreciarse estos fenómenos de simbolización, tanto de los miedos por la inserción de una cárcel en un barrio, como la decepción por las prácticas corruptas, y las esperanzas de reforzar la identidad con los valores patrimoniales. En ello se observa la "percepción transformada en representaciones a través de la imaginación, proceso por el cual la representación sufre una transformación simbólica" (Hiernaux, 2007).

El espacio urbano tiene un rol especial en la activación de la imaginación, debido a que el habitante de la ciudad está constantemente afectado por una avalancha de estímulos visuales, de tensión permanente para la percepción (Simmel, 2015). Como consecuencia, esta fuerte estimulación visual conduce a tres actitudes: de aversión u odio, de libertad o idealidad, y de indiferencia (Vernik, y otros, 2016). De acuerdo con estas observaciones, se clasificaron los resultados sobre el pasado, presente y futuro, como se relacionan a continuación [los detalles de la encuesta se encuentran en el Anexo 1].

\section{Sobre el pasado}

Frente al grado de relación con el inmueble, se halló que el $80 \%$ de los encuestados conocen el panóptico y el $76 \%$ conoce su antiguo uso carcelario, pero el $60 \%$ desconoce por qué se denomina panóptico, o cuál es su significado, en lo que se identifica una debilidad en la socialización y promoción de su valor como tipología arquitectónica, lo cual conduce a una apropiación del objeto pero no del contenido. A ello se suma que el 88\% de los encuestados desconocen en qué año se construyó el inmueble, y el 56\% ignora que el Panóptico fue declarado Monumento Nacional y Bien de Interés Cultural. Estas cifras se relacionan directamente con el 57\% de encuestados que nunca han ingresado a la edificación, a pesar de que a partir de 2018 se realizan actividades en las plazoletas de acceso público.

Estas características se derivan de la falta de comunicación entre las entidades político-administrativas y la población en general, debido a que, tanto el proceso de declaratoria (Alcázar, 1987), como el concurso para realizar el proyecto para su intervención, se realizaron "desde arriba" (Moreno, Burbano, Fonseca, Mancera, \& Arcos, 2010), lo cual fue fundamental para la protección legal del inmueble y la asignación de recursos para su restauración, pero escaso para asegurar la apropiación social del inmueble y la gestión social del proyecto. Estas características son evidentes en la concepción del proyecto de intervención de panóptico, cuyos criterios son exclusivamente materiales y funcionales, sin que existan evidencias de 
mediación y construcción participativa con la comunidad (Saravia Ríos, 2007; Compañía de Diseño Arquitectónico y de Construcción, 2010).

Sobre el presente

En relación al presente del inmueble, el 67\% desconoce su uso actual, el 71\% ignora los planes de restauración que se han llevado a cabo, el 66\% desconoce que desde 2015 se vienen realizando actividades y eventos en las zonas externas del Panóptico de Ibagué y el 65\% no sabe que se han invertido más de 10.000 millones (U\$ 3’090.234) en su recuperación. De ello se desprenden varias consideraciones, como que el desconocimiento es una de las estrategias de manipulación y control, canalizado a través de la indiferencia y evidente en la ausencia de estrategias de sensibilización sobre el patrimonio cultural, que contribuyen a que gran parte de la ciudadanía desconozca o ignore las actividades del proyecto y, más aún, que no se sientan identificados con el Bien de Interés Cultural. Estos resultados se ven reflejados mayoritariamente en niños y jóvenes, y se acrecientan en las comunas distantes al Panóptico.

Como consecuencia, el 62\% de los encuestados considera que el Panóptico ha sido poco o nada importante como elemento significativo de la identidad local y, por lo tanto, es considerado un símbolo de corrupción que despoja a la ciudad de motivos de orgullo. En su reemplazo, emergen otros edificios como íconos de esa identidad, tales como el teatro Tolima, el parque Murillo Toro, la plaza de Bolívar y la catedral, sin que en esa valoración medie la condición de Monumento Nacional. Estos resultados corresponden absolutamente con la consideración de los encuestados frente a que los principales símbolos de la identidad local son la música (63\%), las festividades folclóricas (56\%) y la gastronomía (43,7\%), en comparación con el $3,3 \%$ que considera la arquitectura como parte de su identidad.

En cuanto a la identificación de responsabilidades, el 39\% culpa a la administración local, el 24\% a la gobernación del Tolima, el 20 al Gobierno Nacional y al Ministerio de Cultura y el 15\% a la sociedad en general. Estos resultados afirman la desconfianza en los estamentos públicos en todos los niveles, y existe una pequeña percepción de que la sociedad tiene algún grado de responsabilidad.

El 51\% cree que el proyecto no se ha terminado por factores de corrupción, el 23\% por falta de voluntad política, 15\% por falta de recursos, y el 11\% le atribuye la responsabilidad a la falta de interés de los ciudadanos. Estos datos refuerzan la imagen negativa y la desconfianza que tienen los ciudadanos frente a los estamentos públicos encargados de la recuperación del Panóptico, ya que entre los que culpan a la corrupción y los que responsabilizan a la clase política suman un 74\%. Resalta también el hecho que el $11 \%$ de los encuestados piensa que también la ciudadanía ha sido en mayor o menor medida culpable, lo que denota cierto grado de autocrítica que es necesaria a la hora de emprender esfuerzos conjuntos para la terminación del proyecto.

Sobre el futuro 


\section{La (des)aparición de los monumentos. Materialidad e imaginarios en el panóptico de Ibagué, Colombia}

De acuerdo a las posibilidades de uso futuro, el 26\% considera que el Panóptico debe ser un Museo de la Música, el 20\% un parque interactivo, 15\% una galería de arte, $13 \%$ dice que debe ser un escenario para la formación y fomento de artistas, $12 \%$ que se destine a eventos culturales, y el 3\% que funcione como museo alusivo a los Derechos Humanos. En este punto parece haber un mayor consenso en las respuestas obtenidas, donde la gran mayoría de los encuestados consideran usos culturales, entendidos como actividades artísticas y de participación comunitaria, para la edificación. Estos resultados deberían ser tenidos en cuenta por las autoridades a la hora de establecer el uso final del Panóptico con el fin de que se ajuste de mejor manera a las expectativas ciudadanas.

De igual forma, se observa que a pesar de la malversación de los recursos invertidos, un $89 \%$ considera que la edificación debe estar abierta al público permanentemente y un $41 \%$ estaría dispuesto a pagar por el ingreso y que esos recursos se destinen a su mantenimiento. Finalmente, el 74\% de los encuestados considera que una vez terminado el Panóptico puede llegar a convertirse en un elemento importante de la identidad de los Ibaguereños.

Este $74 \%$ es la muestra de latencia en la población con respecto a las posibilidades del panóptico una vez se termine su proceso de intervención. Controvierte las propias convicciones de la población encuestada sobre la importancia de los monumentos arquitectónicos como parte de su identidad y conduce al deseo de apropiación de los monumentos como factor de cohesión simbólica (García Canclini, 2007).

\section{En síntesis}

Los datos muestran que el panóptico es percibido como un símbolo de corrupción con escaso aporte a las dinámicas sociales y culturales, como consecuencia de su presencia/aparición física ruinosa. El desconocimiento es tanto una estrategia gubernamental de manipulación mediante el alejamiento de la opinión sobre el inmueble para la toma de decisiones desde arriba, así como un rechazo social por el inmueble como símbolo de corrupción. Sin embargo, existe un reconocimiento latente y mayoritario sobre las posibilidades del bien de interés cultural como elemento de transformación socio-cultural, que requiere de su activación mediante la presión social y la responsabilidad gubernamental y de los entes de control. También, es claro que el edificio no genera una conexión directa con la población, sino a través de su uso, lo que significa que el componente físico del panóptico es un elemento vacío para el cual se requiere un uso que genere apropiación. Por ello, la población expresa en su uso futuro la preferencia por la música, el arte y las actividades lúdicas como elementos conectores que conducirían a la construcción o consenso sobre la identidad. Esto explica la relación entre la aparición física y la desaparición en los imaginarios, mediados por la latencia o ilusión.

\section{Discusión}


Para profundizar en la comprensión del significado de los datos hallados en la encuesta, se abordaron los conceptos asociados y su aplicación al panóptico de Ibagué en su condición de edificio y elemento simbólico. En consecuencia, se presentan a continuación los elementos analíticos correspondientes a la triada identidad-cultura-patrimonio, aplicados en la encuesta. Estos tres conceptos tienen lógicas circulares que se referencian mutuamente a través de los procesos de representación simbólica y enfatizan la urgencia de desarrollar conceptos y acciones sobre el patrimonio alrededor de la construcción social.

\section{Identidad}

La identidad es un conjunto de expresiones de los valores esenciales reconocidos por una comunidad, que pueden referirse a la historia y las vivencias comunes, resultado de las interrelaciones múltiples de carácter dialéctico entre las costumbres, las tradiciones y las transformaciones generadas de acuerdo con los cambios en las formas de vida en cada etapa histórica. Es una forma de autoconciencia del universo de "significados y sentidos, proyectos o propuestas, condiciones y situación real [...] en el andamiaje total del espacio sociocultural y la fuerza social que representa. Es el resultado de la configuración y reconfiguración del espacio sin exclusión" (Chaos Yeras, 2018). El patrimonio cultural se interpreta como preexistencia "independiente de su reconocimiento o valoración" y como reflejo de la vida de la comunidad, su historia e identidad. El patrimonio y la identidad son dinámicos y están condicionados por su mutua retroalimentación (Chaos Yeras, 2018; Alfonso, 2014).

La identidad cultural hace referencia a hechos culturales y de conciencia que se manifiestan en las expresiones de vivencias cotidianas de los pueblos y de sus expresiones más concretas, como el lenguaje, las instituciones sociales, la cultura popular, el arte, la literatura y la arquitectura, las cuales cambian en relación con los momentos históricos y las interpretaciones que coexisten en el marco de las relaciones inter generacionales. El patrimonio cultural cuenta como uno de sus elementos fundamentales con el patrimonio arquitectónico, irremplazable expresión de la riqueza y diversidad de cultura de un pueblo (Chaos Yeras, 2018). "La identidad, es una relación dialógica entre la realidad, las ideas y los valores, y los intereses de quienes las propugnan y las comparten", el patrimonio existe por "las representaciones simbólicas de estas versiones de identidad" (Prats, 2000).

La escala de valores derivada de la encuesta, presenta la negación de la importancia del panóptico en la construcción de identidad local, en lo cual se observa una manifestación de rechazo a ser en relación con la ruina arquitectónica, en tanto símbolo de corrupción. Reconocerse en el panóptico, equivaldría a identificarse, o pertenecer a las dinámicas de irregularidad administrativa, en las que solo el 11\% reconoce parte de la responsabilidad. En este caso, la identidad es también una negación, es decir, es algo que no toda la gente tiene, busca, construye ni negocia (Brubaker \& Cooper, 2001, pág. 2). 


\section{La (des)aparición de los monumentos. Materialidad e imaginarios en el panóptico de Ibagué, Colombia}

La relación de la identidad con el concepto de territorio, se establece como un diálogo entre el ser humano y su contexto, para encontrar los vínculos que lo unen con él en el habitar, y cómo, en este sentido, la memoria colectiva ha encontrado en el espacio su realización plena en cuanto a recurso para el recuerdo (Zusman, 2002). La exclusión del espacio de la cárcel desde su concepción, y la exclusión posterior por el estado ruinoso, han impedido una construcción simbólica de reconocimiento y apropiación del patrimonio que significa el panóptico, principalmente porque su sustento se encuentra en su valoración excluyente como patrimonio material.

Las formas del pasado condicionan las acciones, las representaciones y la producción de formas presentes y futuras. Esta «inercia dinámica» es la que permite entonces que «el pasado esté presente». En este cuadro del análisis aparece la metáfora de la «rugosidad», adoptada de la perspectiva geomorfológica de Jean Tricart. Las rugosidades serían herencias morfológicas de carácter socio-geográfico de tiempos pasados (Santos, 1996, pág. 36). Por un lado, ellas resumen la convivencia de testimonios de diferentes momentos históricos, que resisten o se adaptan a nuevas funciones. Ello nos hablaría de una disociación entre forma y contenido.

\section{Cultura}

Consideramos la cultura como un sistema de creencias, valores, normas, símbolos y prácticas colectivas aprendidas y compartidas por los miembros de una colectividad, que constituyen el marco de sus relaciones sociales (Mercado Maldonado \& Hernandez Olivia, 2010, pág. 13). Ello implica que los miembros de cada sociedad generan un conjunto de máximas, a partir de las cuales dan sentido a sus acciones e interpretan los acontecimientos de la vida diaria. De ahí que se diga que la cultura es "el medio en el cual los individuos se forman y del cual extraen las claves y contenidos explicativos, así como el instrumental descodificador, interpretativo y valorativo que les permite interactuar con el resto de las personas que integran o comparten tal cultura" (Piqueras Infante, 1996, pág. 108).

Sin embargo, la cultura, o las culturas, son realidades sistemáticas y cambiantes, que no se pueden abarcar en su totalidad ni detener en el tiempo, de modo que su conocimiento estará determinado por los intereses utilitarios y presentistas, y este es el patrimonio que se puede conservar, comunicar y transmitir (Prats, 2000).

La connotación específica en las encuestas, presenta una sociedad cuyas máximas o consensos de cultura se encuentran en la música (63\%), las festividades folclóricas (56\%) y la gastronomía (43,7\%). La condición espacial de estas máximas, equivale a un $25 \%$ en los paisajes y a un 3,3\% en la arquitectura, en lo cual se advierte que el medio explicativo, interpretativo y valorativo, prescinde del espacio físico urbano/arquitectónico para dar sentido a sus acciones. En el caso del panóptico esta observación es fundamental porque la concepción de conservación del bien de interés cultural se centra en las condiciones materiales del edificio, sin que medie una construcción social de las implicaciones del panóptico como fenómeno urbano con mayores implicaciones que la "materialidad [...] que da soporte a los elementos 
portadores de sentido y, agregaríamos, de identidad y memoria colectiva" (Lacarrieu, 2007).

\section{Patrimonio}

El concepto de patrimonio cultural se encuentra íntimamente ligado a los procesos de construcción de la identidad colectiva, de la apropiación que realizan las sociedades de su propia historia (García Canclini, 1989). El patrimonio cultural expresa la solidaridad que une a quienes comparten un conjunto de bienes y prácticas que los identifican. La apropiación de este capital está mediada por las condiciones y capacidades de relacionarse con el patrimonio, en dependencia de la desigualdad de participación de los grupos sociales (García Canclini, 1999).

El amplio abanico de los elementos que se intersecan en la concepción sobre el patrimonio, y la imposibilidad para abarcarlas completamente, conducen a la consideración del patrimonio como conocimiento de los valores que sustentan las identidades, las culturas. Su interpretación, significación, gestión y promoción, se ejerce entonces mediante un consenso, al que se denomina identidad, en relación con las agendas de quienes lo promuevan (Prats, 2000).

Durante las últimas tres décadas, los planes y visiones de desarrollo de Ibagué han pretendido establecer, desde lo público, diferentes planteamientos en torno a cuál debería ser el elemento o símbolo local. Conceptos como ciudad musical, logística, turística, cultural o universitaria, han aparecido y desaparecido del debate (Secretaría de Planeación Municipal de Ibagué, 2014). Esto evidencia una falta de consenso en torno a los elementos, símbolos o distintivos de Ibagué, y la ambigüedad del concepto de identidad en las agendas promovidas por las entidades gubernamentales.

Dentro de los intentos por determinar y establecer los elementos de la identidad local desde la perspectiva estatal, generalmente se acude a la música como elemento caracterizador, debido a la denominación tradicional como ciudad musical de Colombia y, más recientemente, como la Capital Musical. Como consecuencia, las actividades con mayor impacto y recordación son el Festival Nacional de Música, el Concurso Nacional de Duetos, el Festival Folclórico Colombiano, los encuentros internacionales de piano, violín, guitarra y diversos festivales de géneros musicales como el jazz, rock y música clásica. De ello se deriva la institucionalización de la marca "Ibagué Capital Musical" (Concejo Municipal de Ibagué, 2010), mediante la cual se ha buscado la identificación de los ciudadanos con el símbolo gráfico y conceptual, así como la proyección de la ciudad en el ámbito nacional e internacional.

\section{La identidad musical}

Los orígenes de la identidad musical de Ibagué se encuentran en el libro Viajes a Través de América del Sur, en el que Jean Alexis Cadoine, bajo el pseudónimo de Conde de Gabriac, plasmó los relatos de su travesía por el continente desde 1866 hasta 1867 (Cuartas Coymat Á. , 1994). Gabriac registró cómo la música era un 


\section{La (des)aparición de los monumentos. Materialidad e imaginarios en el panóptico de Ibagué, Colombia}

suceso cotidiano, arraigado en el alma de los ibaguereños, resaltando las manifestaciones coreo-musicales, serenatas de guitarras y flautas, grupos de instrumentistas, variedad de músicos y bailarines que tocaban en las calles y frente a los balcones de las casas (Cuartas Coymat A. , 2013).

El relato de Gabriac contiene elementos fundamentales para el análisis del significado del término ciudad musical, pues indica que sus habitantes mostraban habilidades musicales particulares, tomando en cuenta que no existían academias para la enseñanza de la música, ni escenarios para su difusión. Sin embargo, en cada casa había una persona que interpretaba algún instrumento y las calles abundaban en serenatas nocturnas. La ciudad era la academia y el escenario para la difusión de la música. Como si la música fuera el espíritu de la ciudad, a pesar de los aparentes impedimentos para su desarrollo.

A principios del siglo XX (1906-1909) se creó el conservatorio musical del Tolima (Pardo, 2003), con lo cual inicia el proceso de formalización de las dinámicas observadas por Gabriac. Hacia 1932 se construye la sala de conciertos Alberto Castilla y se emprende la realización de encuentros nacionales de música (Gil Araque, 2009). Este sigue siendo el único escenario creado específicamente para la realización de eventos musicales. Sin embargo, su enfoque académico limita el alcance a nivel de identidad general. Existen otros escenarios multipropósito como el teatro Tolima, la concha acústica del parque Centenario, el parque Murillo Toro o el colegio Champagnat, que son adecuados para diversos eventos, de lo cual se puede deducir la preservación de algunas de las características tradicionales de la condición de Ibagué como ciudad musical, a pesar de la inacción gubernamental y empresarial.

Esta relación deficiente entre el espíritu musical y la infraestructura, o los escenarios para su difusión y desarrollo, muestra claramente que los fenómenos arquitectónicos y urbanísticos han sido inferiores a las necesidades y los retos culturales planteados por el crecimiento musical de Ibagué. La arquitectura está desconectada de la música y, si la música es el corazón de la identidad local, se comprende que el grado de relación con la arquitectura como símbolo de identidad, represente un escaso 3,3\% en las encuestas [Anexo 1]. Si a ello se suman las connotaciones de percepción de corrupción, el estado ruinoso del panóptico y el rechazo hacia el uso de las instalaciones carcelarias, se obtiene una mínima relación de los ciudadanos con el inmueble.

Es también fundamental reconocer que el proyecto de museo de los derechos humanos y la paz, es coherente con el proceso de pos acuerdos en Colombia, entendido como el conjunto de estrategias para la construcción de una paz estable y duradera, derivada de los acuerdos entre el gobierno y las Fuerzas Armadas Revolucionarias de Colombia (FARC) para el fin del conflicto armado (Gobierno Nacional; FARC-EP, 2016). Sin embargo, es consecuencia de una agenda política frente a las dinámicas del parlamento andino, que no indagó por los elementos esenciales de la identidad local o no buscó su armonización en una construcción desde abajo (Carvajal Sánchez, 2011). La encuesta realizada en esta investigación, 


\section{Fabián Andrés Sánchez Rojas - Andrés Ernesto Francel Delgado}

determina claramente que los elementos de identidad de la ciudad que reconoce la población, son la música, el folclor y la gastronomía, sin que exista una producción infraestructural vinculada a estas actividades. Sin embargo, la interpretación sobre el apelativo de ciudad musical, presenta la preservación de las características observadas por Gabriac en el siglo XIX.

El proyecto de intervención del panóptico de Ibagué, pudo partir de una indagación sobre los valores identitarios locales para determinar su uso y relación con la ciudadanía, además de verificar los requerimientos infraestructurales para reforzar el concepto de Capital Musical. La música pervive como elemento de identidad fundamental en los habitantes de la ciudad, sin que los hechos arquitectónicos la refuercen, en lo cual se nota un distanciamiento entre la acción gubernamental y los valores que reconocen los encuestados.

La institucionalización de la marca Ibagué Capital Musical como condensador del festival folclórico, de las actividades gastronómicas y los eventos musicales, sigue la dinámica tradicional observada por Gabriac: la música existe, mueve la ciudad inmaterial, pero el aspecto físico desaparece, o no ha aparecido suficientemente.

La construcción de la identidad local se ha centrado en la emisión de normas para la protección y difusión de los valores musicales, folclóricos y gastronómicos (Concejo Municipal de Ibagué, 2003), instalados en la memoria colectiva, entendida como una corriente de pensamiento continua, que retiene del pasado sólo lo que aún está vivo o es capaz de vivir en la conciencia del grupo que la mantiene (Halbwachs, 1995).

En el marco de este estudio, se comprende que el panóptico tiene un enorme valor simbólico que, sin embargo, no es autónomo para los habitantes de Ibagué, sino en relación con los factores que componen la construcción de un consenso sobre la identidad local. El edificio aparece como un monumento en la ciudad por la gestión de algunos expertos en arquitectura, quienes resaltan la importancia del componente físico, espacial e histórico del inmueble. Sin embargo, la corrupción y la débil relación entre los factores de identidad cultural y el proyecto de intervención del panóptico, han conducido a su desaparición del edificio del presente identitario de Ibagué.

\section{Conclusiones}

La exploración de la triada identidad-cultura-patrimonio en el panóptico de Ibagué, conduce a resaltar la importancia de abordar la intervención de los edificios patrimoniales desde los valores culturales para incorporar los componentes de identidad y evitar que la condición de un bien de interés cultural se reduzca a su componente material, como se observa en el caso del Panóptico. En estas intervenciones es relevante la arbitrariedad, como parte de un sistema de ejercicio de poder y mecanismo de dominación. Es urgente comprender que el acceso público a los dispositivos patrimoniales no es directamente proporcional a los grados de 


\section{La (des)aparición de los monumentos. Materialidad e imaginarios en el panóptico de Ibagué, Colombia}

apropiación que una sociedad realice sobre ellos, mediados por las barreras y exclusiones subyacentes a las dinámicas sociales.

Esta desarticulación entre los componentes materiales e inmateriales del patrimonio, y la ausencia de exploraciones sobre las dimensiones contemporáneas más complejas del sentido del patrimonio, tienen una relación directa con las propuestas de los planes de ordenamiento territorial, que carecen de elementos de definición sobre las características de la ciudad, y reducen lo que debería ser una carta de navegación y ejecución de proyectos, a un formalismo que se modifica incesantemente en dependencia de los diversos intereses particulares y las agendas políticas que confluyen en las dinámicas urbanas.

La consecuencia de estos procesos de desarticulación es la dicotomía recurrente entre los proyectos de patrimonio como entidades materiales y la ausencia de los vínculos sociales que lo legitiman. Esta fragmentación del concepto de patrimonio se reitera en la condición de que los principales elementos de la cultura local no tienen una representación material, lo que significa una persistencia de las representaciones simbólicas de la cultura en los imaginarios colectivos y una ausencia de dispositivos arquitectónicos y urbanísticos que los potencien. De ello se desprende que los imaginarios se transformen hacia una repulsión por el panóptico en la medida en que se arruina la pieza arquitectónica. La intervención del panóptico ignora los valores de la identidad, pero aparecen los valores del patrimonio material, degradados por la ineficiencia gubernamental. Lograr su conjunción, es el principal

reto que se debe asumir en el futuro.

\section{Bibliografía}

Alcaldía municipal de Ibagué. (25 de julio de 2004). Decreto 0431. Por medio del cual se hace un reconocimiento público al Parlamento Andino. Ibagué, Colombia. Obtenido

de https://www.ibague.gov.co/portal/admin/archivos/normatividad/2004/DECRET 0\%20431.PDF

Alcázar, J. A. (1987). Proyecto de declaración como monumento nacional del edificio del panóptico de Ibagué. Ibagué: Instituto tolimense de cultura.

Alfonso, A. (2014). Conservación del patrimonio cultural inmueble. Temas introductorios. La Habana, Cuba: Editorial Universitaria Félix Varela.

Arango, S. (1989). Historia de la arquitectura en Colombia. Bogotá D.C.: Centro editorial y Facultad de Artes, Universidad Nacional de Colombia.

Brubaker, R., \& Cooper, F. (2001). Mas allá de la identidad. Buenos Aires : Revista de Apuntes de Investigación del CECyP $\mathrm{N}^{\circ} 7$. 
Calero Polanco, Jessica Tatiana; García, Catalina. (25 de septiembre de 2014). El Panóptico de Ibagué: un museo en el papel. El Nuevo Día. Obtenido de http://www.elnuevodia.com.co/nuevodia/especiales/sucesos/233101-elpanoptico-de-ibague-un-museo-en-el-papel

Carvajal Sánchez, N. I. (2011). Enfoques ascendente y descendente del ordenamiento territorial en Colombia. Cuadernos de Geografía-Revista Colombiana de Geografía, 20(1), 21-28. doi:https://doi.org/10.15446/rcdg.v20n1.23064

Chaos Yeras, M. T. (março de 2018). Usos y apropiaciones del patrimonio urbano vs. identidad perdida o conservada. PatryTer. Revista Latinoamericana e Caribenha de $\begin{array}{llll}\text { Geografía } e & \text { Humanidades, } & \text { 22-33. }\end{array}$ doi:https://doi.org/10.26512/patryter.v1i1.7108

Cifuentes Segovia, M. F. (julio-diciembre de 2015). Retrospectiva constructiva e histórica del Panóptico de Ibagué, Colombia. Apuntes, 28(2), 16-29. doi:http://dx.doi.org/10.11144/Javeriana.apc28-2.rchp

Compañía de Diseño Arquitectónico y de Construcción. (2010). Www.codiarco.com. Obtenido de http://www.codiarco.com/intervencion/Panptico-de-Ibague

Concejo Municipal de Ibagué. (19 de junio de 2003). Decreto 0503. Por medio del cual se crea la orden del tamal. Ibagué. Obtenido de https://www.ibague.gov.co/portal/admin/archivos/normatividad/2003/DECRET 0\%20503.PDF

Concejo Municipal de Ibagué. (4 de octubre de 2010). Acuerdo 26 de 2010. Por medio del cual se adopta oficialmente la marca - Ibagué capital musical - como logotipo institucional del municipio de Ibagué. Ibagué. Obtenido de http://www.concejodeibague.gov.co/documentos_2009/2010/Acuerdo026_2010. pdf

Concejo Municipal de Ibagué. (17 de agosto de 2011). Acuerdo 018 de 2011. Por medio del cual se crea el sistema municipal de planeación y presupuesto participativo del desarrollo de Ibagué y se dictan otras disposiciones. Ibagué, Tolima, Colombia. Obtenido de http://www.concejodeibague.gov.co/documentos_2009/2011/Acuerdo018_2011. pdf

Concejo Municipal de Ibagué. (27 de diciembre de 2018). Acuerdo 034 de 2018. Por el cual se modifica la estructura organizacional de la alcaldía municipal de Ibagué, se definen las funciones de sus dependencias y sedictan otras disposiciones. Ibagué, Tolima, Colombia. Obtenido de https://www.ibague.gov.co/portal/admin/archivos/normatividad/2019/22833ACU-20190103.PDF 


\section{La (des)aparición de los monumentos. Materialidad e imaginarios en el panóptico de Ibagué, Colombia}

Creswell, J. W. (2008). Mixed Methods Research: State od Art. Michigan, EEUU: University of Michigan.

Cuartas Coymat, Á. (1994). Ibagué ciudad histórica. Ibagué: Pjao Editores.

Cuartas Coymat, A. (2013). El Conde de Gabriac en Ibagué. Ibagué: Alcaldía Municipal de Ibagué.

Departamento Administrativo Nacional de Estadística DANE. (2016). Estimación y Proyección de Población Nacional, Departamental y Municipal Total por Área 1985 - 2020. Bogotá D.C.: DANE.

Durand, G. (1994). L'imaginaire. Essai sur les sciences et la philosophie de l'image. París: Hatier.

Ecos del Combeima. (25 de abril de 2019). La Alcaldía de Ibagué reconoce que están retrasadas las obras del Panóptico. Ecos del Combeima, pág. Archivo digital de noticias. Obtenido de http://www.ecosdelcombeima.com/ibague/nota-141864-laalcaldia-de-ibague-reconoce-que-estan-retrasadas-las-obras-del-panoptico

El Nuevo Día. (1 de marzo de 2015). Curaduría urbana abre la vía para urbanizar granja San Jorge. EL Nuevo Día. Recuperado el 2 de octubre de 2015, de http://www.elnuevodia.com.co/nuevodia/tolima/ibague/249326-curaduriaurbana-uno-da-via-libre-a-urbanizar-en-la-granja-san-jorge

El Tiempo. (3 de diciembre de 2010). Las iglesias de Arturo Jaramillo Concha en Bogotá EI Tiempo. Obtenido de http://www.eltiempo.com/colombia/bogota/ARTICULO-WEBNEW_NOTA_INTERIOR-8517053.html

Erickson, E. (1977). La identidad psicosocial. En Enciclopedia Internacional de las Ciencias Sociales. Tomo V(págs. 586-591). España: Aguilar.

Físcalia General de la Nación secional Tolima. (18 de julio de 2012). Implicados por "elefante blanco" del panóptico. Ecos del Combeima, pág. Archivo digital de noticias. Obtenido de http://www.ecosdelcombeima.com/judiciales/nota-19381implicados-elefante-blanco-del-panoptico

Foucault, M. (2002). Vigilar y Castigar. Nacimiento de la prisión. Buenos Aires: Siglo XXI Editores Argentina S.A.

Francel, A. (2015). Belén: una mujer, un barrio, una historia. Ibagué, 1920-1925. Ibagué: Caza de Libros Editores. Obtenido de https://www.researchgate.net/publication/323916561_Belen_una_mujer_un_barr io_una_historia_Ibague_1920-1926 
Francel, A. (2015). Metodología y reflexiones para la reconstrucción de la historia urbana del barrio Belén de Ibagué (1921-1925). V Simposio Colombiano de Historia Regional y Local HistoReLo (págs. 1-13). Ibagué: Universidad Nacional de Colombia. Obtenido de https://www.researchgate.net/publication/332353240_METODOLOGIA_Y_REFLE XIONES_PARA_LA_RECONSTRUCCION_DE_LA_HISTORIA_URBANA_DEL_BARRIO_B ELEN_DE_IBAGUE_1921-1925

Francel, A., \& Ojeda, J. A. (Septiembre-diciembre de 2016). Conceptual and stylistic architecture interactions in the Republican period. Ibagué, Colombia, 1893-1945. Arquitectura y Urbanismo, XXXVII(3), 1-19. Recuperado el 15 de enero de 2016, de http://rau.cujae.edu.cu/index.php/revistaau/article/view/379

García Canclini, N. (13-16 de junio de 1989). ¿Quiénes usan el patrimonio? Políticas culturales y participación social. Ponencia presentada en las Jornadas Taller: EI Uso del Pasado. Obtenido de http://s3.amazonaws.com/academia.edu.documents/31732059/39740485Canclini-

Quienesusanelpatrimonio.pdf?AWSAccessKeyId=AKIAJ56TQJRTWSMTNPEA\&Expi res $=1471476228 \&$ Signature $=$ aHLUrYEwBY\%2FxmAyfEg04p1uR0zc\%3D\&respon se-content-disposition=inline $\% 3 \mathrm{~B} \% 20$ filename $\% 3 \mathrm{D} 397$

García Canclini, N. (1999). Los usos sociales del patrimonio cultural. En E. Aguilar Criado, Patrimonio Etnológico. Nuevas perspectivas de estudio (págs. 16-33). Junta de Andalucía, Instituto Andaluz del Patrimonio Histórico. Obtenido de http://bibliotecadigital.academia.cl/jspui/bitstream/123456789/617/1/Nestor\% 20Garcia\%20Canclini.pdf

García Canclini, N. (agosto de 2007). Diálogo con Néstor García Canclini: ¿qué son los imaginarios y cómo actúan en la ciudad? Revista eure, 89-99. (A. Lindón, Entrevistador) Santiago de Chile: volumen 33, número 99. Obtenido de https://repositorio.uc.cl/bitstream/handle/11534/8129/000485577.pdf?sequenc $\mathrm{e}=1$

Gil Araque, F. (noviembre de 2009). Congresos Nacionales de la Música 1936-1937. Revista Música, cultura y pensamiento, 1(1), 13-34.

Gómez, A. (mayo de 2015). Los barrios parque y de fin de semana en el mapa de la expansión metropolitana. Buenos Aires, 1910-1950. EURE, 159-185. Obtenido de http://www.scielo.cl/pdf/eure/v41n123/art07.pdf

Gravano, A. (2005). Palimpsesto urbano Sobre-escritura de huellas diacrónicas de la ciudad imaginada. En S. Boggi, C. Caffarelli, A. Gravano, M. C. Leiro, \& P. Zamora, Imaginarios sociales de la ciudad media. Emblemas, fragmentaciones y otredades urbanas. Estudios de Antropología Urbana (págs. 27-40). Tandil-Olavarría: 


\section{La (des)aparición de los monumentos. Materialidad e imaginarios en el panóptico de Ibagué, Colombia}

Universidad Nacional del Centro de la Provincia de Buenos Aires, Red de Editoriales de Universidades Nacionales. Obtenido de https://s3.amazonaws.com/academia.edu.documents/55209638/Libro_Imaginari osCiudadMedia_ARIEL_GRAVANO_compilador.pdf?response-contentdisposition=inline $\% 3 B \% 20$ filename\%3DLibro_ImaginariosCiudadMedia_ARIEL_G RAVA.pdf\&X-Amz-Algorithm=AWS4-HMAC-SHA256\&X-Amz-Cred

Halbwachs, M. (1995). Memoria colectiva y memoria histórica. Reis. Revista Española de Investigaciones Sociológicas(69), 209-219. Obtenido de http://ih-vmcisreis.c.mad.interhost.com/REIS/PDF/REIS_069_12.pdf

Hernández Sampieri, R. (2006). Metodología de la Investigación 4a Edición. México D.F.: McGraw-Hill.

Hiernaux, D. (2006). Los centros históricos: ¿espacios posmodernos?(De choques de imaginarios y otros conflictos). En A. Lindón, D. Hiernaux, \& M. Á. Aguilar, Lugares e imaginarios en la metrópolis (págs. 27-41). Barcelona: Anthropos Editorial. Rubí (Barcelona). División de Ciencias Sociales y Humanidades. Universidad Autónoma Metropolitana, Iztapalapa, México.

Hiernaux, D. (agosto de 2007). Los imaginarios urbanos: de la teoría y los aterrizajes en los estudios urbanos. Revista eure, 33(99), 17-30. doi:http://dx.doi.org/10.4067/S0250-71612007000200003

Isaza Nieto, P. (24 de mayo de 2015). El panóptico de Ibagué. El Nuevo Día, pág. Versión digital. Obtenido de http://www.elnuevodia.com.co/nuevodia/opinion/columnistas/258289-elpanoptico-de-ibague

Izasa Nieto, P. (24 de mayo de 2015). El Panóptico de Ibagué. El Nuevo Día, pág. Archivo digital de noticias. Recuperado el 5 de junio de 2018, de http://www.elnuevodia.com.co/nuevodia/opinion/columnistas/258289-elpanoptico-de-ibague

Krippendorff, K. (1990). Metodología de Análisis de Contenido: Teoría y Práctica. Barcelona: Paidós.

Lacarrieu, M. (agosto de 2007). La "insoportable levedad" de lo urbano. Revista eure, 33(99), 47-64. doi:http://dx.doi.org/10.4067/S0250-71612007000200005

Lindón, A. (agosto de 2007). La ciudad y la ida urbana a través de los imaginarios urbanos. Revista eure, 33(99), 7-16. doi:http://dx.doi.org/10.4067/S025071612007000200002 
Mejía Navarrete, J. (2000). El Muestreo en la Investigación Científica. Lima: Revista de Investigaciones Sociales.

Mercado Maldonado, A., \& Hernandez Olivia, A. V. (2010). El Proceso de Construcción de la Identidad Colectiva. México: Universidad Autónoma del Estado de México.

Mertens, D. M. (2005). Research and Evaluation in Education and Psychology: Integrating Diversity Whit Quantitative, and Mixed Methods 2a Edición. Madrid: SAGE Publications.

Ministro de Cultura de Colombia. (30 de julio de 1998). Resolucion numero 0752. Por la cual se declara como Bien de Interés Cultural de Carácter Nacional algunos inmuebles localizados en Ibagué - Tolima, Medellín y Titiribí - Antioquia y Santa Fe de Bogotá. Santa Fe de Bogotá, D. C.: Diario Oficial de Colombia. Obtenido de https://2019.vlex.com/\#WW/vid/59802096

Moreno, O., Burbano, D., Fonseca, L., Mancera, M., \& Arcos, B. (2010). El quehacer del arquitecto javeriano. (B. Arcos, Ed.) Bogotá: Pontificia Universidad Javeriana. Obtenido de https://issuu.com/margaritamancera/docs/_primera_muestra_del_ejercicio_profe sional_

Pardo, C. O. (2003). La educación en Ibagué. En D. Ramírez, \& \&. otros, Compendio de historia de Ibagué. Ibagué: Academia de historia del Tolima, Gobernación del Tolima, Alcaldía de Ibagué.

Pedraza Vargas, L. M., Urueña Silva, D. C., \& Zuluaga Duque, E. (2018). Mantenimiento y rehabilitación de la infraestructura paisajista del panóptico de la ciudad de Ibagué departamento del Tolima. Ibagué: Universidad Cooperativa de Colombia. Trabajo de grado para la obtención del titulo de Ingeniero civil. Obtenido de http://repository.ucc.edu.co/handle/ucc/4933

Pieto Páez, L., Arango, S., Ramírez Nieto, J., Colón, L., Império, L., Delgadillo, H., \& Carrasco Zaldúa, F. (2017). Alberto Manrique Martin. Bogotá: Universidad Nacional de Colombia, Sociedad Colombiana de Arquitectos, Alcaldía Mayor, Instituto Distrital de Patrimonio Cultural. Obtenido de https://issuu.com/patrimoniobogota/docs/alberto_manrique_mart_n

Piqueras Infante, A. (1996). La identidad valenciana. La difícil construcción de una identidad colectiva. Madrid: Institució Valenciana D’estudios I Investigació .

Prats, L. (2000). El concepto de patrimonio cultural. Cuadernos de Antopología Social(11), 115-136. Obtenido de http://revistascientificas.filo.uba.ar/index.php/CAS/article/view/4709/4206 


\section{La (des)aparición de los monumentos. Materialidad e imaginarios en el panóptico de Ibagué, Colombia}

Pulido García, A. M., \& Lovera Bernal, A. F. (2018). Parque Panóptico : distrito cultural de enseñanza, artes y oficios del Tolima. Bogotá: Universidad de La Salle. Obtenido de http://repository.lasalle.edu.co/handle/10185/29145

Redacción El Tiempo. (3 de junio de 2003). Panóptico será una realidad. El Tiempo, pág. Archivo digital de noticias. Recuperado el 12 de 06 de 2019, de https://www.eltiempo.com/archivo/documento/MAM-1017384

Restrepo, J. (17 de junio de 2008). Antigua cárcel penitenciaria de Ibagué se convertirá en museo de los Derechos Humanos. El Tiempo, pág. Archivo digital de noticias. Obtenido de https://www.eltiempo.com/archivo/documento/CMS4302329

Rivera, O. (2012). Preso en el olvido: El Panóptico De Ibagué. Revista Ergoletrías(1), 66-68. Obtenido

de http://revistas.ut.edu.co/index.php/ergoletrias/article/view/1536

Santos, M. (1996). A natureza do espaço. Técnica e tempo. Razão e emoção. Sao Paulo: Hucitec.

Saravia Ríos, E. (23 de agosto de 2007). Museo panóptico. Revista Arcadia Semana(23), pág. 32 Obtenido de https://books.google.com.co/books?id=GGYEAAAAMBAJ\&pg=PT30\&lpg=PT30\&d $\mathrm{q}=$ luis +humberto+duque+gomez+ganador+pan\%C3\%B3ptico+ibagu $\% \mathrm{C} 3 \% \mathrm{~A} 9 \&$ so urce $=$ bl\&ots $=$ Qk8ATE7IQy\&sig=ACfU3U2Dcn-PBJRSk-

VGyc2Pbwj0GX3e0w\&hl=es\&sa=X\&ved=2ahUKEwiQ4_eFnb_lAhWiq1kKHWUiCuQ Q6AEwA3oECAkQ

Secretaría de Planeación Municipal de Ibagué. (2014). Archivo planes de desarrollo municipales. Ibagué: Alcaldía de Ibagué.

Silva, A. (2006). Imaginarios Urbanos. Bogotá, Colombia: Arango Editores, Ltda.

Simmel, G. (2015). El secreto y las sociedades secretas. (S. Muller, Trad.) Madrid: Ediciones Sequitur.

Trujillo Muñoz, A. (segundo semestre de 2007). Alberto Castilla, más allá de la música. Revista Aquelarre, volumen 6, número 12. , 6(12), 28-34.

UNESCO. (2015). Patrimonio Mundial. Paris: UNESCO.

Vallejo, Y. (26 de julio de 2018). Panóptico, de elefante blanco a promotor del turismo cultural. Ecos del Combeima, pág. Archivo digital de noticias. Obtenido de http://www.ecosdelcombeima.com/actualidad/nota-129994-panoptico-deelefante-blanco-promotor-del-turismo-cultural 
Vernik, E., Hoffmann, T. S., Bohn, C., Borisonik, H., García Chicote, F., Jung, W., . . . Wegelin, L. (2016). Visiones de la libertad. Acerca de la cuestión central en Simmel. (E. Vernik, \& H. Borisonik, Edits.) Buenos Aires: Instituto de Investigaciones Gino Germani - UBA. Obtenido de http://biblioteca.clacso.edu.ar/clacso/se/20170816104717/Georg_Simmel.pdf

Zusman, P. (2002). Milton Santos. Su legado teórico y existencial (1926-2001). Documents d'anàlisi geogràfica(40), 205-222. Obtenido de http://www.raco.cat/index.php/DocumentsAnalisi/article/view/31765/31599

Recibido: 25/10/2019

Evaluado: 28/12/2019

Versión Final: 30/01/2020 